\title{
The difference in blood pressure readings between arms and survival: primary care cohort study
}

\author{
(c) $(1)(9)$ OPEN ACCESS
}

\author{
Christopher E Clark clinical academic fellow ${ }^{1}$, Rod S Taylor professor in health services research, \\ medical statistician ${ }^{1}$, Angela C Shore professor of cardiovascular science ${ }^{2}$, John L Campbell \\ professor of general practice and primary care ${ }^{1}$
}

${ }^{1}$ Primary Care Research Group, Institute of Health Services Research, Peninsula College of Medicine and Dentistry, University of Exeter, Devon EX1 2LU, UK; ${ }^{2}$ Institute of Biomedical and Clinical Science, Peninsula College of Medicine and Dentistry, and Peninsula NIHR Clinical Research Facility, University of Exeter, Devon, UK

\begin{abstract}
Objective To determine whether a difference in systolic blood pressure readings between arms can predict a reduced event free survival after 10 years.

Design Cohort study.

Setting Rural general practice in Devon, United Kingdom.

Participants 230 people receiving treatment for hypertension in primary care.

Intervention Bilateral blood pressure measurements recorded at three successive surgery attendances.

Main outcome measures Cardiovascular events and deaths from all causes during a median follow-up of 9.8 years.

Results At recruitment 24\% (55/230) of participants had a mean interarm difference in systolic blood pressure of $10 \mathrm{~mm} \mathrm{Hg}$ or more and $9 \%$ $(21 / 230)$ of $15 \mathrm{~mm} \mathrm{Hg}$ or more; these differences were associated with an increased risk of all cause mortality (adjusted hazard ratio 3.6, 95\% confidence interval 2.0 to 6.5 and $3.1,1.6$ to 6.0 , respectively). The risk of death was also increased in 183 participants without pre-existing cardiovascular disease with an interarm difference in systolic blood pressure of $10 \mathrm{~mm} \mathrm{Hg}$ or more or $15 \mathrm{~mm} \mathrm{Hg}$ or more (2.6, 1.4 to 4.8 and $2.7,1.3$ to 5.4). An interarm difference in diastolic blood pressure of $10 \mathrm{~mm} \mathrm{Hg}$ or more was weakly associated with an increased risk of cardiovascular events or death.
\end{abstract}

Conclusions Differences in systolic blood pressure between arms can predict an increased risk of cardiovascular events and all cause mortality over 10 years in people with hypertension. This difference could be a valuable indicator of increased cardiovascular risk. Bilateral blood pressure measurements should become a routine part of cardiovascular assessment in primary care.

\section{Introduction}

A difference in blood pressure readings between arms can be observed in various general populations, ${ }^{12}$ healthy women during the antenatal period, ${ }^{34}$ and populations with an increased risk of cardiovascular disease, such as people with hypertension, ${ }^{5}$ diabetes, ${ }^{67}$ chronic renal disease, ${ }^{8}$ or peripheral vascular disease. ${ }^{9}$ The presence of a difference between arm measurements has been implicated in a delayed diagnosis of hypertension ${ }^{10}$ and is associated with a higher prevalence of poor control in hypertension, ${ }^{9}$ as failure to standardise measurement to the arm with the highest reading can mislead decisions about management. Current guidelines recognise the need to check blood pressure in both arms ${ }^{11}{ }^{12}$ but this practice has not been followed by many clinicians, ${ }^{13}{ }^{14}$ including general practitioners in the United Kingdom, ${ }^{15}$ and continues to be undertaken selectively in primary care settings. ${ }^{16}$ This poor uptake may in part result from a lack of clearly presented supporting evidence for this intervention that is relevant to primary care practitioners. ${ }^{17}$

The new clinical guideline for hypertension from the National Institute for Health and clinical Excellence considers an interarm difference of less than $10 \mathrm{~mm} \mathrm{Hg}$ to be normal and attributes a difference of more than $20 \mathrm{~mm} \mathrm{Hg}$ to underlying vascular disease. ${ }^{18}$ An evidence review for interarm differences in blood pressure was outside the remit of this update; however, it did not consider the group with an interarm difference in systolic blood pressure of 10-20 mm Hg, despite our previous systematic review suggesting that this group represents $15 \%$ of the population with hypertension. ${ }^{1}$

Our previous work has suggested that an interarm difference in blood pressure is an independent predictor of reduced event free 
survival. ${ }^{5}$ Others have also shown an association with increased mortality in people at higher risk of vascular disease, ${ }^{89}$ and the need for further studies carried out in primary care settings has been highlighted. ${ }^{9}$ We further analysed an established primary care cohort to determine to what extent the difference in survival observed at five years persists at 10 years. ${ }^{5}$

\section{Methods}

The Mid Devon Medical Practice operates three separate surgeries in a rural area. This study was carried out in the main surgery (list size 1900). Eligible participants were those registered with the author (CEC) and receiving treatment for hypertension. Hypertension was defined by the then current guidelines of the British Hypertension Society $(\geq 160 / 100 \mathrm{~mm}$ $\mathrm{Hg}$ or $\geq 140 / 90 \mathrm{~mm} \mathrm{Hg}$ in the presence of target organ damage, diabetes, or coronary heart disease risk score $\geq 15 \%) .{ }^{20} \mathrm{We}$ excluded participants on the basis of anatomical criteria (loss, previous injury, surgery above wrist level, or paralysis of one arm) or for practical reasons - that is, the inability or unwillingness to regularly attend the surgery for review.

\section{Measurements}

At recruitment one investigator (CEC) measured blood pressure using a standard mercury sphygmomanometer (Accoson; AC Cossor, Harlow, Essex), which was calibrated every six months. Standard or large cuffs were used as appropriate. Pairs of blood pressure readings were collected sequentially after the participant had been seated for five minutes; measurement was taken in the arm first presented without prompting, and the cuff was then swapped to the other arm and another measurement taken. The arm was supported during each measurement. We obtained single pairs of measurements at the first and subsequent two visits and recruitment ran from 9 November 1999 to 17 June 2002. Return visits were planned every six months if blood pressure was controlled, or at shorter intervals if treatment for high blood pressure was being adjusted. We averaged the three pairs of readings to obtain a mean systolic and diastolic blood pressure for each arm to derive the mean interarm difference. After the first visit we recorded the participant's medical history and characteristics (age, sex, smoking status, body mass index, glucose level, total cholesterol level, creatinine level, pre-treatment blood pressure, years since diagnosis of hypertension, evidence of left ventricular hypertrophy on electrocardiogram, and Framingham risk score calculated from pre-treatment values extracted from patient records). If such data were missing we undertook the necessary investigations. Drugs were adjusted to achieve optimal blood pressure control according to guidelines, but we did not include use of drugs in the dataset. ${ }^{20}$ We prospectively collected data on events until 26 April 2011. Events were defined as death (cardiovascular or all cause using death certification data augmented where available by post mortem findings) or non-fatal cerebrovascular events, and cardiovascular events (myocardial infarction or a new diagnosis of angina), confirmed after referral to secondary care.

\section{Data analysis}

We entered anonymised data on an Excel spreadsheet and used SPSS Predictive Analytics Software Statistics v18.0.0 and Stata v11.1 for analysis. Left ventricular hypertrophy was diagnosed by electrocardiography at recruitment using the Perugia scoring system, ${ }^{21}$ and we calculated scores for the risk of coronary heart disease at 10 years from the Framingham equation. ${ }^{22} \mathrm{We}$ compared the participant's characteristics at entry to the cohorts using $t$ or $\chi^{2}$ tests between groups according to predefined cut-off points of interarm differences that have been used in the literature and in our previous report-namely, $10 \mathrm{~mm} \mathrm{Hg}$ or more and $15 \mathrm{~mm} \mathrm{Hg}$ or more differences in systolic blood pressure and $10 \mathrm{~mm} \mathrm{Hg}$ or more differences in diastolic blood pressure. At each of these cut-off points we used Kaplan-Meier survival plots to compare the time to death (all cause and cardiovascular), combined non-fatal cardiovascular and cerebrovascular events, and death or non-fatal events. We used a Cox's proportional hazards regression model to calculate the unadjusted hazard ratios for these outcomes, fitting interarm difference as either a continuous variable or using the defined cut-off points. A multivariable Cox regression model was used to derive adjusted hazard ratios, which included the Framingham risk score (model 1) and, in addition, included mean blood pressure (systolic for analyses of systolic interarm difference, and diastolic for analyses of diastolic interarm difference, calculated as the mean of three pairs $(n=6)$ of blood pressure measurements at recruitment), presence of diabetes, and pre-existing cardiovascular or peripheral vascular disease on entry to the cohort (model 2). Regardless of their statistical effect we included these variables in the model for their relevance on clinical grounds. To assess the specific contribution of interarm differences in blood pressure, we used the likelihood ratio test to assess the reduction in goodness of fit arising as the result of omitting the interarm difference term from each of the adjusted models. A predefined secondary analysis was undertaken in the subgroup of participants without previous cardiovascular disease at entry to the cohort. We assessed proportionality of hazards over time by plotting $-\ln (-\ln ($ survival $))$ versus $\ln ($ analysis time $)$, and tested this using Schoenfeld residuals. ${ }^{23}{ }^{24}$ (Also see web extra on bmj.com.) We found no major violations of the proportional hazards assumption. The competing risk of death was accounted for by censoring at the date of death. In mortality outcome models, we considered any previous non-fatal events to be uninformative.

\section{Results}

Of 273 patients ( $14.3 \%$ of surgery list) eligible for inclusion, $247(90 \%)$ were recruited by June 2002 and 230 (84\%) with complete data were analysed (fig $1 \Downarrow$ ). No participants were lost to follow-up. Participants not included in the analysis were significantly older but did not differ in any other respect from the cohort analysed (table $1 \Downarrow$ ). The median time to collection of all three sets of readings was four months (interquartile range 2-12 months). At recruitment, 55 (24\%) participants had a mean interarm difference in systolic blood pressure of $10 \mathrm{~mm} \mathrm{Hg}$ or more and $21(9 \%)$ a difference of $15 \mathrm{~mm} \mathrm{Hg}$ or more (fig $2 \Downarrow$ ); $14(6 \%)$ participants had an interarm difference in diastolic blood pressure of $10 \mathrm{~mm} \mathrm{Hg}$ or more. The mean difference in systolic blood pressure was $1.5 \mathrm{~mm} \mathrm{Hg}$ (95\% confidence interval 0.4 to 2.6) higher in the right arm and in diastolic blood pressure was $1.7 \mathrm{~mm} \mathrm{Hg}$ (1.1 to 2.3 ) higher in the left arm.

The median time to final analysis or to a fatal event was 9.8 years (interquartile range 0.4-11.4 years). During the study period, 52 cardiovascular and 27 cerebrovascular events occurred in 76 participants. Fifty nine participants died and a total of $100(44 \%)$ participants had an event or died.

\section{All case analysis}

There were no differences in the baseline characteristics of participants above and below an interarm difference in systolic blood pressure of $10 \mathrm{~mm} \mathrm{Hg}$ or more or $15 \mathrm{~mm} \mathrm{Hg}$ or more or 
an interarm difference in diastolic blood pressure of $10 \mathrm{~mm} \mathrm{Hg}$ or more (table $2 \Downarrow$ ).

In both unadjusted and adjusted analyses an interarm difference in systolic blood pressure of $10 \mathrm{~mm} \mathrm{Hg}$ or more and $15 \mathrm{~mm} \mathrm{Hg}$ or more were both associated with increases in the hazard of cardiovascular events, cardiovascular mortality, all cause mortality, and combined non-fatal events or all cause mortality. Hazard ratios for all cause mortality after full adjustment were 3.6 (95\% confidence interval 2.0 to 6.5 ) for interarm differences in systolic blood pressure of $10 \mathrm{~mm} \mathrm{Hg}$ or more (fig $3 \Downarrow$ ) and 3.1 (1.6 to 6.0) for differences of $15 \mathrm{~mm} \mathrm{Hg}$ or more (table $3 \Downarrow$ ). This corresponded to a 5-6\% increase in the hazard of outcomes for each $1 \mathrm{~mm} \mathrm{Hg}$ increment in interarm difference for systolic blood pressure (table $4 \Downarrow$ ). An interarm difference in diastolic blood pressure of $10 \mathrm{~mm} \mathrm{Hg}$ or more was associated, with less precision, with increases in the hazard of cardiovascular events and combined non-fatal events or all cause mortality in both unadjusted and adjusted analyses: adjusted hazard ratios 3.8 (95\% confidence interval 1.8 to 8.6 ) and 3.3 (1.6 to 6.8), respectively. After adjustment this corresponded to a $9 \%$ increase in hazard of any fatal or non-fatal event for each $1 \mathrm{~mm}$ $\mathrm{Hg}$ increment in interarm difference for diastolic blood pressure. Across all models for systolic blood pressure the likelihood ratio test showed a significant reduction in goodness of fit on removal of interarm difference, with the exception of cardiovascular mortality associated with a difference of $15 \mathrm{~mm}$ $\mathrm{Hg}$ or more, indicating that inclusion of interarm differences in systolic blood pressure consistently improved the predictability of models. This was also shown for an interarm difference in diastolic blood pressure of $10 \mathrm{~mm} \mathrm{Hg}$ or more with non fatal events and combined events and deaths (tables 3 and 4).

\section{Analyses without pre-existing cardiovascular disease}

At recruitment 183 participants had no pre-existing cardiovascular disease; $28 \%(\mathrm{n}=51)$ had an interarm difference in systolic blood pressure of $10 \mathrm{~mm} \mathrm{Hg}$ or more and $11 \%(\mathrm{n}=20)$ a difference of $15 \mathrm{~mm} \mathrm{Hg}$ or more, and $6 \%(\mathrm{n}=11)$ had an interarm difference in diastolic blood pressure of $10 \mathrm{~mm} \mathrm{Hg}$ or more. In this subgroup, sensitivity analyses of predefined levels of interarm differences in both systolic and diastolic blood pressures were examined for differences in mortality and event free survival. The findings of unadjusted and adjusted Cox regression analyses were consistent with the full case analysis, showing increases in the hazards of cardiovascular events, cardiovascular mortality, all cause mortality, and combined events or deaths in both unadjusted and adjusted analyses for interarm differences in systolic blood pressure of $10 \mathrm{~mm} \mathrm{Hg}$ or more or $15 \mathrm{~mm} \mathrm{Hg}$ or more; hazard ratios for all cause mortality were 2.6 (95\% confidence interval 1.4 to 4.8 ; fig $4 \Downarrow)$ and 2.7 (1.3 to 5.4). An interarm difference in diastolic blood pressure of $10 \mathrm{~mm} \mathrm{Hg}$ or more was associated with an increased hazard of cardiovascular events, and combined non-fatal events or all cause mortality after adjustment: hazard ratios 3.2 (1.3 to 8.1) and 2.4 (1.0 to 5.9); table $5 \Downarrow$. When interarm difference was analysed as a continuous variable, the hazard of all outcomes increased by a consistent $5 \%$ for each $1 \mathrm{~mm} \mathrm{Hg}$ increment in interarm difference in systolic blood pressure after adjustment. In the continuous model the hazard ratios for an interarm difference in diastolic blood pressure were no longer significant (table 4). Associated reductions in goodness of fit were seen on removal of interarm differences in systolic blood pressure from the models.

\section{Stratified analysis}

In primary prevention of cardiovascular disease a 10 year cardiovascular risk score of more than $20 \%$ over 10 years represents a threshold for intervention with statin therapy. ${ }^{25}$ Participants without pre-existing cardiovascular disease were therefore stratified by both their conventional risk score (above or below the threshold of 20\%) and their interarm difference in systolic blood pressure (above or below $10 \mathrm{~mm} \mathrm{Hg}$ ). Analysis showed that the presence of an interarm difference in systolic blood pressure of $10 \mathrm{~mm} \mathrm{Hg}$ or more without pre-existing disease, but where the cardiovascular risk score was more than $20 \%$, was associated with a significantly higher hazard of combined fatal and non-fatal events compared with those with an interarm difference in systolic blood pressure of less than 10 $\mathrm{mm} \mathrm{Hg}$ but equivalent cardiovascular risk score (log rank statistic $25.9, \mathrm{P}<0.001$ ) and also a higher hazard when compared with those with pre-existing disease (log rank statistic 4.5, $\mathrm{P}<0.05$; fig $5 \Downarrow)$.

\section{Discussion}

A difference in systolic blood pressure between arms of $10 \mathrm{~mm}$ $\mathrm{Hg}$ or more or $15 \mathrm{~mm} \mathrm{Hg}$ or more in people with hypertension in primary care was associated with a reduction in event free survival over 10 years. This association held for the study population when participants with pre-existing cardiovascular disease were included or excluded and, compared with our earlier analysis at 4.7 years' follow-up, the survival curves have continued to diverge. The presence of an interarm difference in systolic blood pressure of $10 \mathrm{~mm} \mathrm{Hg}$ or more in participants not known to have cardiovascular disease at recruitment but with a high cardiovascular risk score of more than $20 \%$ at baseline, seems to confer a level of risk for events of similar magnitude to that of participants with previously diagnosed cardiovascular disease.

\section{Strengths and limitations of the study}

One investigator (CEC) gathered all the data. In designing this study, a sequential method of measurement was chosen as a pragmatic test within the consultation. A recent meta-analysis has suggested that a simultaneous, automated repeated measurement method with one or two machines should be the ideal for epidemiological study, ${ }^{26}$ and we also, subsequent to establishing this study, showed that a simultaneous measurement technique reduces bias. ${ }^{1}$ Studies relevant to the general population using such techniques ${ }^{27-31}$ have reported lower prevalences of an interarm difference in blood pressure than those using sequential measurements. ${ }^{2} 1929{ }^{22-38}$ However, subsequent sampling of the study cohort presented here, using a robust simultaneous measurement technique, showed similar prevalence rates for interarm differences of $10 \mathrm{~mm} \mathrm{Hg}$ or more of $19 \%$ for systolic blood pressure and $7 \%$ for diastolic blood pressure (compared with the $23 \%$ and $6 \%$ reported here). ${ }^{39}$ One study also reported a strong correlation of interarm differences when comparing three simultaneous and three sequentially gathered pairs of readings, and a correlation remained when measurements were repeated at a later date. ${ }^{40} \mathrm{We}$ have presented data suggesting that a single sequential pair of measurements can reliably rule out an interarm difference in blood pressure with high negative predictive value, ${ }^{41}$ and our new meta-analysis has shown no difference in the strength of association between peripheral vascular disease and systolic interarm differences in blood pressure according to the method of measurement. ${ }^{42}$ Given current knowledge, if designing this study now we would adopt an automated simultaneous measurement method, although we 
believe that our pragmatic approach remains relevant to "real world" practice. ${ }^{43}$

The lack of strict randomisation of the order of arm measurement could have introduced bias, as blood pressure measurements will often decrease during repetition. ${ }^{11}$ No strict first arm preference was adopted, however, and the small absolute difference in blood pressure in favour of right or left arms is in keeping with previously reported large series and suggests that no systematic bias was introduced. ${ }^{19} 27244$ The investigator CEC was not blinded to data collection, but because events were recorded prospectively over 10 years, and our definitions of non-fatal events required independent diagnosis in secondary care, we do not believe that this lack of blinding could have biased the survival outcomes reported. We did not collect data on drug use at recruitment or changes in use during the study period, so cannot comment on potential survival differences due to drugs.

This is a small study from one rural general practice. The reported prevalence of participants receiving treatment for hypertension in this study was $14.3 \%$ of the list, comparable to the $13.7 \%$ for women and $11.7 \%$ for men reported at the time by the health survey for England. ${ }^{45}$ Therefore we believe that these findings can be generalised to other similar cohorts of people with hypertension being treated in primary care, although the lack of representation of ethnic minority groups in Devon is a recognised limitation. Conversely, this rural practice has a low turnover of patients, facilitating long term follow-up, which we see as a strength.

Based on the previous analysis of this cohort, giving a hazard ratio of 2.5 for the composite outcome of mortality or event at a cut-off point for interarm difference in systolic blood pressure of $10 \mathrm{~mm} \mathrm{Hg}$, we estimated that we would have required a total of 50 observed events to achieve $90 \%$ power and 62 to achieve $95 \%$ power. In this follow-up we observed 108 deaths or events. ${ }^{1}$ Higher cut-off values were initially included in the analysis plan to permit comparison with other survival studies ${ }^{8}$; however, the diminishing numbers of participants with an interarm difference in systolic blood pressure of more than $15 \mathrm{~mm} \mathrm{Hg}$ meant a reduced precision of the event results for higher interarm differences, these analyses are therefore not included in the data presented.

\section{Comparison with existing literature}

Prevalence of an interarm difference in blood pressure varies with the population studied and tends to be higher in the presence of hypertension. ${ }^{29}{ }^{37} 46$ Whether this reflects a truly higher prevalence or is merely a function of higher achievement of an arbitrary cut-off point where absolute values are increased is unclear, but the prevalence values reported here are consistent with our previous meta-analysis of similar cohorts measured with robust techniques.

Some previous series have suggested a bias towards higher readings from the right arm, ${ }^{2} 3133$ 36-38 4748 whereas others have failed to show this ${ }^{192732444649}$; studies looking specifically at left or right handedness have also failed to show an association with the arm producing the higher reading..$^{291}$ This study has not found a consistent bias to one side for interarm differences in either systolic or diastolic blood pressures, and shows that measurement using both arms at initial assessment is required to avoid future underestimation of blood pressure owing to an unrecognised difference between arms. In fact the direction of mean differences in systolic and diastolic blood pressures was opposite, in keeping with some other reported series, and we suggest this may be related to differing pulse pressures along the aorta. ${ }^{31}{ }^{50}$ Current guidance suggests that an interarm difference of less than $10 \mathrm{~mm} \mathrm{Hg}$ can be considered to be normal and that differences of $20 / 10 \mathrm{~mm} \mathrm{Hg}$ or more warrant specialist referral. ${ }^{18}$ Previous studies have proposed a normal range for differences in systolic or diastolic blood pressure of up to 10 $\mathrm{mm} \mathrm{Hg}^{27}$ and it has been argued from other cross sectional series that the high prevalence of differences above these cut-off points implies that this is a physiological rather than a pathological finding. ${ }^{450}$ Our recent meta-analysis has, however, shown that an interarm difference in systolic blood pressure of $10 \mathrm{~mm} \mathrm{Hg}$ or more or $15 \mathrm{~mm} \mathrm{Hg}$ or more is associated with peripheral vascular disease in cross sectional studies, with a pooled risk ratio of 2.4 or 2.5 , respectively. ${ }^{42}$ Peripheral vascular disease is recognised as a risk factor for future cardiovascular events and mortality, ${ }^{52}$ and we have previously proposed that an interarm difference in blood pressure is due to peripheral vascular disease. ${ }^{53}$ An interarm difference in systolic blood pressure of $15 \mathrm{~mm} \mathrm{Hg}$ or more is associated with angiographic evidence of carotid or aortic artery disease, ${ }^{54}$ and vascular disease of the arms is associated with hypertension. ${ }^{55}$ Thus evidence supports the association of an interarm difference with existing peripheral vascular disease, and we propose that this accounts for the survival differences seen.

Two other prospective studies have reported increased mortality with an interarm difference in systolic blood pressure; the larger study reported a significant survival difference with a difference of $15 \mathrm{~mm} \mathrm{Hg}$ or more. Three cohorts were combined in that paper and differences were not significant for the cohort recruited from the community; only total deaths were significantly higher in one of the two cohorts recruited from vascular clinics. ${ }^{9}$ The other study, of renal and general medical out-patients, showed significantly higher rates of events or death for an interarm difference in systolic blood pressure of $10 \mathrm{~mm}$ $\mathrm{Hg}$ or more. ${ }^{8}$ Meta-analysis to combine data for an interarm difference in systolic blood pressure of $15 \mathrm{~mm} \mathrm{Hg}$ or more presented here with three other cohorts ${ }^{9}$ was possible and showed pooled hazard ratios for all cause mortality of $1.6(95 \%$ confidence interval 1.1 to 2.3) and for cardiovascular related mortality of 1.7 (1.1 to 2.5 ). ${ }^{42}$ Thus there is supporting evidence from other studies that an interarm difference in blood pressure is a predictor of cardiovascular events and death in populations at high baseline cardiovascular risk.

\section{Implications for clinical practice}

Guidelines advocate the measurement of blood pressure in both arms as part of the initial assessment of hypertension. ${ }^{11}{ }^{12}$ Failure to recognise a difference can lead to underestimation or under-treatment of blood pressure, but this guidance is not routinely followed in primary care ${ }^{13-15}$ and the additional time required to measure both arms has been cited as a practical problem. ${ }^{656}$ It is postulated that this poor uptake may be due in part to a lack of evidence thought to be relevant by primary care practitioners, ${ }^{17}$ and guidelines should make use of available evidence rather than consensus views where evidence does exist. ${ }^{42}$ The finding here of an additional cardiovascular risk associated with an interarm difference in a representative primary care cohort with hypertension is therefore important. In this study, regression modelling showed the interarm difference to be an independent variable associated with increased risk of events or death, and in stratified analysis its presence conferred a risk equivalent to established cardiovascular disease. This would suggest that those with an interarm difference may justifiably require aggressive management along secondary prevention guidelines, such as the addition of statin therapy, and potentially by adding 
antiplatelet treatment to their care; a strategy that remains of unclear benefit in primary cardiovascular prevention. ${ }^{57}$ This seems a logical and pathologically plausible proposal since such treatment is effective in secondary prevention and therefore ought to be effective in primary prevention for selected cases at the highest vascular risk; however, further work is needed to justify this approach. We have suggested that the interarm difference is a manifestation of occult peripheral vascular disease ${ }^{53}$ which is known to confer an increased risk of events or death ${ }^{58}{ }^{59}$ and is therefore managed with aggressive lifestyle and drug strategies. Non-invasive studies have repeatedly shown an association of interarm differences in systolic blood pressure of $10 \mathrm{~mm} \mathrm{Hg}$ or more ${ }^{56394960}$ and $15 \mathrm{~mm} \mathrm{Hg}$ or more ${ }^{5639495461-63}$ with peripheral vascular disease, and we consider that confirmation of an interarm difference may indicate that further assessment for the presence of peripheral vascular disease should be undertaken..$^{42}$ To date, however, there are no studies of relevant interventions on which to base clinical recommendations.

\section{Implications for future research}

This study adds important information to the evidence base supporting the need to detect an interarm difference in blood pressure, not only to improve measurement and management of hypertension but to consider the vascular risk to the patient. The interarm difference in blood pressure has the potential to act as a simple non-invasive test, identifying those who could benefit from more intensive assessment - for example, by measurement of ankle-brachial pressure index. This measurement is not routinely undertaken in the primary care assessment of patients with hypertension and is not included in the NHS health check programme. ${ }^{64}$ It requires time, experience, and training ${ }^{65}$ whereas bilateral brachial blood pressure measurements can be easily taken. Therefore, further studies of the association of interarm differences in blood pressure with other manifestations of peripheral and cardiovascular disease, in cohorts representative of the primary care population, would be valuable.

\section{Conclusions}

An interarm difference in systolic blood pressure of $10 \mathrm{~mm} \mathrm{Hg}$ or more or $15 \mathrm{~mm} \mathrm{Hg}$ or more predicts reduced mortality and event free survival over 10 years in people with hypertension in primary care. This study supports the potential value of an interarm difference as a simple clinical indicator of increased cardiovascular risk. Assessment of blood pressure in both arms is recommended by guidelines and should become a core component of initial blood pressure measurement in primary care. Detection of an interarm difference should prompt consideration of further vascular assessment and aggressive management of risk factors.

We thank the staff and patients of the Mid Devon Medical Practice for their support and participation in this study.

Contributors: CEC conceived the study, collected the data, undertook primary analyses, and drafted the manuscript. RST undertook and supervised data analysis and contributed to the manuscript. ACS and JLC advised on data analysis and presentation and contributed to the manuscript. CEC wlll act as guarantor for this study.

Funding: This research was supported by the Scientific Foundation Board of the Royal College of General Practitioners (grant No SFB-2009-06), the South West GP Trust, and the National Institute for Health Research (NIHR) Peninsula Collaboration for Leadership in Applied Health Research and Care, a NIHR funded collaboration of the
Peninsula College of Medicine and Dentistry, University of Exeter, University of Plymouth, and NHS South West. The project was also supported by the NIHR Peninsula Clinical Research Facility. The views and opinions expressed in this paper are those of the authors and not necessarily those of the NHS, the NIHR, or the Department of Health.

Competing interests: All authors have completed the ICMJE uniform disclosure form at www.icmje.org/coi_disclosure.pdf (available on request from the corresponding author) and declare: no support from any organisation for the submitted work; no financial relationships with any organisations that might have an interest in the submitted work in the previous three years; and no other relationships or activities that could appear to have influenced the submitted work.

Ethical approval: This study was approved by the North and East Devon Research Ethics Committee (No 2000/12/158).

Data sharing: No additional data available.

1 Clark CE, Campbell JL, Evans PH, Millward A. Prevalence and clinical implications of the inter-arm blood pressure difference: a systematic review. J Hum Hypertens 2006;20:923-31.

2 Cassidy $\mathrm{P}$, Jones $\mathrm{K}$. A study of inter-arm blood pressure differences in primary care. $J$ Hum Hypertens 2001;15:519-22.

3 Ray WT. Assessment of blood pressure discrepancies in third-trimester hypertensive gravidas. AANA J 2000;68:525-30.

4 Poon LCY, Kametas N, Strobl I, Pachoumi C, Nicolaides KH. Inter-arm blood pressure differences in pregnant women. BJOG 2008:115:1122-30.

5 Clark CE, Powell RJ, Campbell JL. The interarm blood pressure difference as predictor of cardiovascular events in patients with hypertension in primary care: cohort study. $J$ Hum Hypertens 2007;21:633-6.

6 Clark CE, Greaves C, Evans PH, Dickens A, Campbell JL. The interarm blood pressure difference in type 2 diabetes: a barrier to effective management? Br J Gen Pract 2009;59:428-32.

7 Kleefstra N, Houweling ST, Meyboom D, Bilo HJG. Measuring the blood pressure in both arms is of little use; longitudinal study into blood pressure differences between both arms and its reproducibility in patients with diabetes mellitus type 2. Nederlands tijdschrift voor geneeskunde 2007;151:1509-14.

8 Agarwal R, Bunaye Z, Bekele DM. Prognostic significance of between-arm blood pressure differences. Hypertension 2008:51:657-62.

9 Aboyans V, Criqui MH, McDermott MM, Allison MA, Denenberg JO, Shadman R, et al. The vital prognosis of subclavian stenosis. J Am Coll Cardiol 2007;49:1540-5.

10 Banks MJ, Erb N, George P, Pace A, Kitas GD. Hypertension is not a disease of the left arm: a difficult diagnosis of hypertension in Takayasu's arteritis. $J$ Hum Hypertens 2001;15:573-5.

11 Williams B, Poulter NR, Brown MJ, Davis M, McInnes GT, Potter JF, et al. Guidelines for management of hypertension: report of the fourth working party of the British Hypertension Society, 2004-BHS IV. J Hum Hypertens 2004;18:139-85.

12 Mancia G, De Backer G, Dominiczak A, Cifkova R, Fagard R, Germano G, et al. 2007 guidelines for the management of arterial hypertension: the Task Force for the Management of Arterial Hypertension of the European Society of Hypertension (ESH) and of the European Society of Cardiology (ESC). Eur Heart J 2007;28:1462-536.

13 Gosse $\mathrm{P}$. Blood pressure should be measured in both arms on the first consultation [comment]. J Hypertens 2002;20:1045-6.

4 Materson B. Inter-arm blood pressure differences. J Hypertens 2004;22:2267-8.

15 Heneghan C, Perera R, Mant D, Glasziou P. Hypertension guideline recommendations in general practice: awareness, agreement, adoption, and adherence. Br J Gen Pract 2007;57:948-52.

16 Clark CE, Smith LFP, Harding G, Taylor RS, Campbell JL. Nurse led hypertension clinics: evolving ahead of the evidence? J Hum Hypertens 2011;25:S6.

17 Parker E, Glasziou P. Use of evidence in hypertension guidelines: should we measure in both arms? Br J Gen Pract 2009;59:e87-92.

18 National Institute for Health and Clinical Excellence. Hypertension: the clinical management of primary hypertension in adults, CG127. NICE, 2011.

19 Clark CE, Powell RJ. The differential blood pressure sign in general practice: prevalence and prognostic value. Fam Pract 2002;19:439-41.

20 Ramsay L, Williams B, Johnston G, MacGregor G, Poston L, Potter J, et al. Guidelines for management of hypertension: report of the third working party of the British Hypertension Society. J Hum Hypertens 1999;13:569-92.

21 Schillaci G, Verdecchia P, Borgioni C, Ciucci A, Guerrieri M, Zampi I, et al. Improved electrocardiographic diagnosis of left ventricular hypertrophy. Am J Cardiol 1994;74:714-9.

22 Hingorani AD, Vallance P. A simple computer program for guiding management of cardiovascular risk factors and prescribing. BMJ 1999;318:101-5.

23 Collett D. Modelling survival data in medical research. 2nd ed. Chapman \& Hall/CRC 2003.

24 Schoenfeld D. Partial residuals for the proportional hazards regression model. Biometrika 1982;69:239-41.

25 JBS 2: Joint British Societies' guidelines on prevention of cardiovascular disease in clinical practice. Heart 2005;91(suppl 5):v1-52.

26 Verberk WJ, Kessels AGH, Thien T. Blood pressure measurement method and inter-arm differences, a meta-analysis. Am J Hypertens 2011: published online 21 July.

27 Orme S, Ralph SG, Birchall A, Lawson-Matthew P, McLean K, Channer KS. The normal range for inter-arm differences in blood pressure. Age Ageing 1999;28:537-42.

28 Gould BA, Hornung RS, Kieso HA, Altman DG, Raftery EB. Is the blood pressure the same in both arms? Clin Cardiol 1985;8:423-6.

29 Harrison EG, Roth GM, Hines EA. Bilateral indirect and direct arterial pressures. Circulation 1960;22:419-36.

30 Fotherby MD, Panayiotou B, Potter JF. Age-related differences in simultaneous interarm blood pressure measurements. Postgrad Med J 1993;69:194-6. 


\section{What is already known on this topic}

A difference in blood pressure readings between arms is frequently observed in various general populations

An interarm difference in systolic blood pressure has been associated with increased mortality in selected secondary care populations at high vascular risk

\section{What this study adds}

In a primary care cohort with hypertension, an interarm difference in systolic blood pressure of $10 \mathrm{~mm} \mathrm{Hg}$ or more or $15 \mathrm{~mm} \mathrm{Hg}$ or more was associated with an increased risk of cardiovascular and all cause mortality over 10 years

For people with hypertension but no pre-existing cardiovascular disease, an interarm difference in systolic blood pressure seems to confer a level of risk equivalent to that of people with hypertension and pre-existing cardiovascular disease

Blood pressure measurement in both arms should become a routine part of cardiovascular assessment in primary care

31 Lane D, Beevers M, Barnes N, Bourne J, John A, Malins S, et al. Inter-arm differences in blood pressure: when are they clinically significant? J Hypertens 2002;20:1089-95.

32 Singer AJ, Hollander JE. Blood pressure. Assessment of interarm differences. Arch Intern Med 1996;156:2005-8.

33 Kay WE, Gardner KD. Comparative blood pressures in the two arms. Cal West Med 1930;33:578-9.

34 Israel E. Differences in blood pressure in both arms. Acta Med Orientalia 1944:3:86-9.

35 Hashimoto F, Hunt WC, Hardy L. Differences between right and left arm blood pressures in the elderly. West J Med 1984;141:189-92.

36 Rueger MJ. Blood pressure variations in the two arms. Ann Intern Med 1951;35:1023.

37 Amsterdam B, Amsterdam AL. Disparity in blood pressures in both arms in normals and hypertensives and its clinical significance. NY State J Med 1943:43:2294-300.

38 Korns KM, Guinand PH. Inequality of blood pressure in the brachial arteries, with especial reference to disease of the arch of the aorta. J Clin Invest 1933;12:143.

39 Clark CE, Campbell JL, Powell RJ, Thompson JF. The inter-arm blood pressure difference and peripheral vascular disease: cross sectional study. Fam Pract 2007;24:420-6.

40 Eguchi K, Yacoub M, Jhalani J, Gerin W, Schwartz JE, Pickering TG. Consistency of blood pressure differences between the left and right arms. Arch Intern Med 2007;167:388-93.

41 Clark CE, Campbell JL, Evans PH, Shore A, Taylor R. Detection of an interarm blood pressure difference in primary care diabetes care. Diabet Med 2009;26:128.

42 Clark CE, Taylor RS, Shore AC, Ukoumunne OC, Campbell JL. The association of an inter-arm difference in systolic blood pressure with vascular disease and mortality: a systematic review and meta-analysis. Lancet : published online ahead of print $30 \mathrm{Jan}$ 2012. www.thelancet.com/journals/lancet/article/PIIS0140-6736\%2811\%2961710-8/ abstract.

43 Clark CE. Inter-arm blood pressure measurement needs to be practical and accurate. Am J Hypertens 2011;24:1189-90.

44 Pesola GR, Pesola HR, Lin M, Nelson MJ, Westfal RE. The normal difference in bilateral indirect blood pressure recordings in hypertensive individuals. Acad Emerg Med 2002;9:342-5.

45 National Centre for Social Research. Health survey for England 2003. NatCen, 2003.

46 Kristensen BO, Kornerup HJ. Which arm to measure the blood pressure? Acta Med Scand 1982;670:69-73.

47 O'Shea JC, Murphy MB. Ambulatory blood pressure monitoring: which arm? J Hum Hypertens 2000;14:227-30.

48 Southby R. Some clinical observations on blood pressure and their practical application, with special reference to variation of blood pressure readings in the two arms. Med J Aust 1935;2:580.

49 Frank SM, Norris EJ, Christopherson R, Beattie C. Right and left arm blood pressure discrepancies in vascular surgery patients. Anesthesiology 1991;75:457-63.

50 Pesola GR, Pesola HR, Nelson MJ, Westfal RE. The normal difference in bilateral indirect blood pressure recordings in normotensive individuals. Am J Emerg Med 2001:19:43-5.

51 Beevers G, Lip GYH, O'Brien E. ABC of hypertension: blood pressure measurement. BMJ 2001;322:981-5.
52 Ankle Brachial Index Collaboraton. Ankle brachial index combined with Framingham risk score to predict cardiovascular events and mortality: a meta-analysis. JAMA 2008;300:197-208

53 Clark CE. Difference in blood pressure between arms might reflect peripheral vascular disease. BMJ 2001;323:399-400.

54 Baribeau Y, Westbrook B, Charlesworth D, Hearne M, Bradley W, Maloney C. Brachial gradient in cardiac surgical patients. Circulation 2002;106(suppl 1):111-3.

55 Sin Lau Y, Yeung J, Krishna Lingam M. Vascular disease of the upper limb. Modern Hypertension Management 2005;5:9-12.

56 Anekwe L. Interarm BP disparity increases heart risk. Pulse 2007;10:17.

57 Fowkes FG, Price JF, Stewart MCW, Butcher I, Leng GC, Pell ACH, et al. Aspirin for prevention of cardiovascular events in a general population screened for a low ankle brachial index: a randomized controlled trial. JAMA 2010;303:841-8.

58 Criqui MH, Langer RD, Fronek A, Feigelson HS, Klauber MR, McCann TJ, et al. Mortality over a period of 10 years in patients with peripheral arterial disease. $N$ Engl J Med 1992;326:381-6.

59 Criqui MH, Denenberg JO, Langer RD, Fronek A. The epidemiology of peripheral arterial disease: importance of identifying the population at risk. Vasc Med 1997;2:221-6.

60 Kawamura T. Assessing ankle-brachial index (ABI) by using automated oscillometric devices. Arq Bras Cardiol 2008;90:294-8.

61 Aboyans V, Kamineni A, Allison MA, McDermott MM, Crouse JR, Ni H, et al. The epidemiology of subclavian stenosis and its association with markers of subclinica atherosclerosis: the Multi-Ethnic Study of Atherosclerosis (MESA). Atherosclerosis 2010;211:266-70.

62 Igarashi Y, Chikamori T, Tomiyama H, Usui Y, Hida S, Tanaka H, et al. Clinical significance of inter-arm pressure difference and ankle-brachial pressure index in patients with suspected coronary artery disease. $J$ Cardiol 2007;50:281-9.

63 Shadman R, Criqui MH, Bundens WP, Fronek A, Denenberg JO, Gamst AC, et al. Subclavian artery stenosis: prevalence, risk factors, and association with cardiovascular diseases. J Am Coll Cardiol 2004;44:618-23.

64 Department of Health. Putting prevention first. Vascular checks: risk assessment and management. DoH, 2008.

65 Ray SA, Srodon PD, Taylor RS, Dormandy JA. Reliability of ankle:brachial pressure index measurement by junior doctors. Br J Surg 1994;81:188-90.

Accepted: 13 January 2012

Cite this as: BMJ 2012;344:e1327

This is an open-access article distributed under the terms of the Creative Commons Attribution Non-commercial License, which permits use, distribution, and reproduction in any medium, provided the original work is properly cited, the use is non commercial and is otherwise in compliance with the license. See: http://creativecommons.org/licenses/bync/2.0/ and http://creativecommons.org/licenses/by-nc/2.0/legalcode. 


\section{Tables}

Table 1| Characteristics of people included and excluded from analyses. Values are numbers (percentages) unless stated otherwise Framingham score

Characteristics

Full data $(n=230)$ Incomplete data $(n=17) \quad P$ value

Continuous variables

Mean (SD) age (years)

$68.1(9.6) \quad 79.2(9.8) \quad<0.001^{*}$

Mean (SD) body mass index

$27.9(4.4) \quad 26.9(3.0) \quad 0.26^{*}$

Mean baseline blood pressure $(\mathrm{mm} \mathrm{Hg})$ :

\begin{tabular}{lccc}
\hline Mean (SD) systolic & $160.5(17.0)$ & $165.8(23.4)$ & $0.37^{\star}$ \\
\hline Mean (SD) diastolic & $86.1(8.6)$ & $89.5(8.6)$ & $0.13^{\star}$
\end{tabular}

Mean (SD) diast

$6.1(8.6)$

$5(29)$

$0.17 \dagger$

Men $107(47)$

$0(0)$

$0.62 \ddagger$

Pre-existing ischaemic heart disease or peripheral vascular disease

$18(8)$

$0(0)$

Smoker

$32(14)$

$4(24)$ $0.76 \ddagger$

Deaths (all causes)

$59(26)$

$4(18)$

Events or deaths

$100(44)$

$7(41)$
$0.40 \ddagger$

${ }^{\star} t$ test.

tx $x^{2}$ test.

‡Fisher's exact test. 
Table 2/ Comparison of baseline variables and outcome measures for interarm differences in systolic blood pressure and in diastolic blood pressure. Values are numbers (percentages) unless stated otherwise

\begin{tabular}{|c|c|c|c|c|c|c|c|c|c|}
\hline \multirow[b]{2}{*}{ Variables } & \multicolumn{6}{|c|}{ Interarm difference in systolic blood pressure $(\mathrm{mm} \mathrm{Hg})$} & \multicolumn{3}{|c|}{$\begin{array}{l}\text { Interarm difference in diastolic blood pressure ( } \mathrm{mm} \\
\mathrm{Hg})\end{array}$} \\
\hline & $<10(n=175)$ & $\geq 10(n=55)$ & $P$ value & $<15(n=209)$ & $\geq 15(n=21)$ & $P$ value & $<10(n=216)$ & $\geq 10(n=14)$ & $P$ value \\
\hline \multicolumn{10}{|l|}{ Continuous variables } \\
\hline Mean (SD) age (years) & $67.6(9.4)$ & $69.7(10.1)$ & $0.16^{*}$ & $67.9(9.4)$ & $70.7(11.5)$ & $0.28^{*}$ & $68.2(9.7)$ & $66.8(7.2)$ & $0.49^{*}$ \\
\hline $\begin{array}{l}\text { Mean (SD) body mass } \\
\text { index }\end{array}$ & $28.0(4.6)$ & $27.3(3.6)$ & $0.23^{*}$ & $27.9(4.4)$ & $27.4(4.2)$ & $0.60^{*}$ & $27.9(4.5)$ & $26.9(2.7)$ & $0.23^{*}$ \\
\hline
\end{tabular}

Mean baseline blood

pressure $(\mathrm{mm} \mathrm{Hg})$ :

\begin{tabular}{|c|c|c|c|c|c|c|c|c|c|}
\hline Systolic blood pressure & $160.1(17.2)$ & $161.8(16.3)$ & $0.51^{*}$ & $160.2(16.8)$ & $163.3(19.2)$ & $0.44^{*}$ & $160.1(16.5)$ & $167.1(23.1)$ & $0.28^{*}$ \\
\hline Diastolic blood pressure & $86.1(8.7)$ & $86.1(8.2)$ & $0.99^{*}$ & $86.1(8.8)$ & $86.1(6.0)$ & $0.98^{*}$ & $85.8(8.3)$ & $90.6(12.0)$ & $0.17^{*}$ \\
\hline $\begin{array}{l}\text { Framingham } 10 \text { year risk } \\
\text { score }(\%)\end{array}$ & $24.9(12.1)$ & $28.4(11.7)$ & $0.058^{*}$ & $25.5(12.1)$ & $28.4(12.1)$ & $0.31^{*}$ & $25.8(12.1)$ & $24.7(11.9)$ & $0.75^{\star}$ \\
\hline \multicolumn{10}{|l|}{ Dichotomous variables } \\
\hline Male & $81(46)$ & $26(47)$ & $0.90 \dagger$ & $98(47)$ & $9(43)$ & $0.72 \dagger$ & $100(46)$ & $7(50)$ & $0.79+$ \\
\hline Diabetes & $12(7)$ & $6(11)$ & $0.39 \ddagger$ & $17(8)$ & $1(5)$ & $1.00 \ddagger$ & $18(8)$ & $0(0)$ & $0.61 \ddagger$ \\
\hline Angina & $25(14)$ & $4(7)$ & $0.17 \dagger$ & $28(13)$ & $1(5)$ & $0.49 \ddagger$ & $28(13)$ & $1(3)$ & $1.00 \ddagger$ \\
\hline Myocardial infarction & $17(10)$ & $2(4)$ & $0.26 \ddagger$ & $19(9)$ & $0(0)$ & $0.23 \ddagger$ & $17(8)$ & $2(14)$ & $0.33 \ddagger$ \\
\hline Cerebrovascular disease & $12(7)$ & $5(9)$ & $0.73 \dagger$ & $14(7)$ & $3(14)$ & $0.43 \dagger$ & $17(8)$ & $0(0)$ & $0.53 \dagger$ \\
\hline $\begin{array}{l}\text { Peripheral vascular } \\
\text { disease }\end{array}$ & $5(3)$ & $0(0)$ & $0.34 \ddagger$ & $5(2)$ & $0(0)$ & $1.00 \ddagger$ & $4(2)$ & $1(7)$ & $0.27 \ddagger$ \\
\hline Smoker & $23(13)$ & $9(16)$ & $0.55 \dagger$ & $28(13)$ & $4(19)$ & $0.51 \neq$ & $30(14)$ & $2(14)$ & $1.00 \ddagger$ \\
\hline
\end{tabular}

${ }^{*} t$ test.

$+x^{2}$ test.

‡Fisher's exact test. 


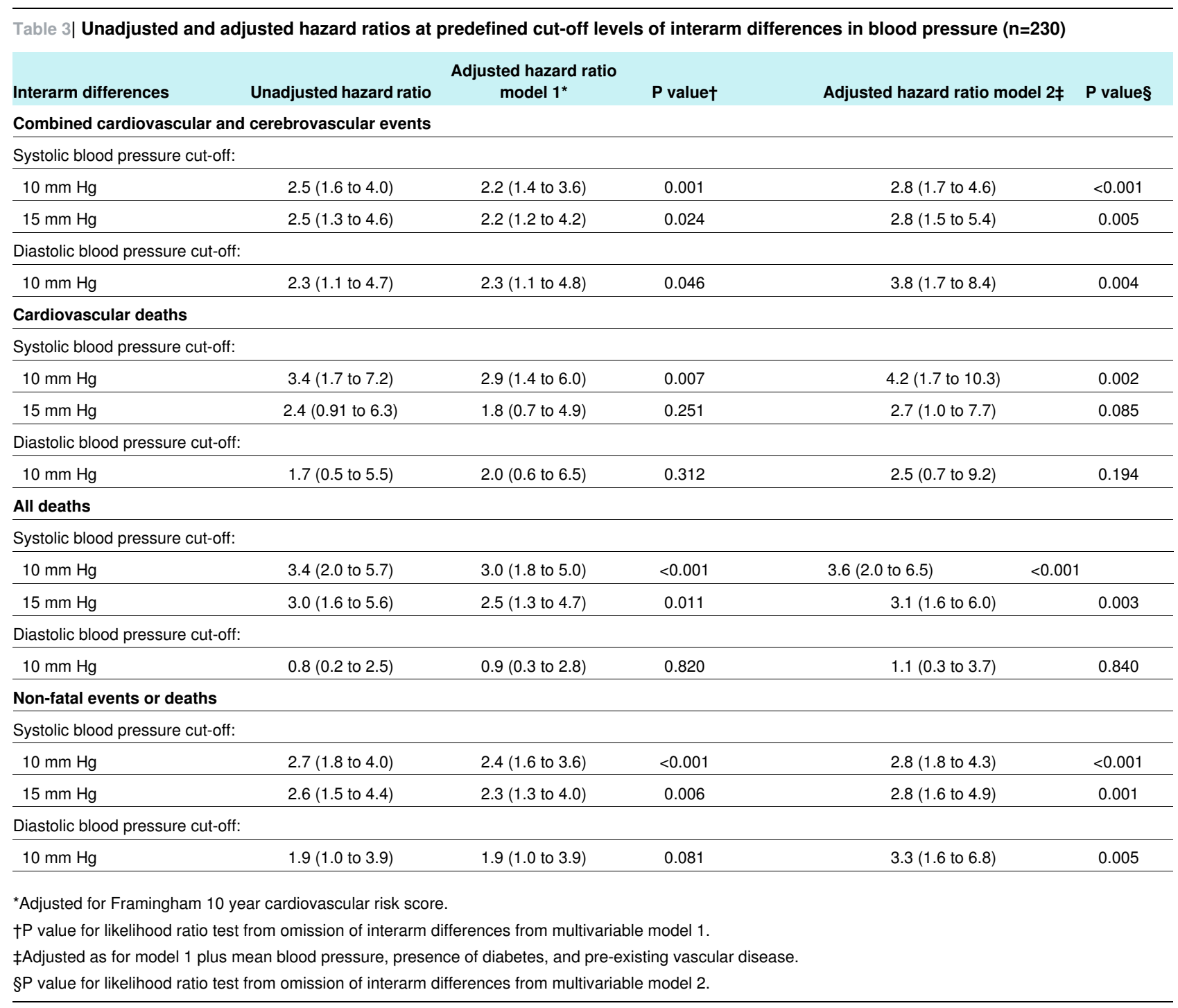


Table 4| Unadjusted and adjusted hazard ratios for absolute interarm differences in systolic and diastolic blood pressure (risk per mm $\mathrm{Hg}$ difference)

\begin{tabular}{|c|c|c|c|c|c|}
\hline Interarm differences & Unadjusted hazard ratio & $\begin{array}{l}\text { Adjusted hazard ratio } \\
\text { model } 1^{*}\end{array}$ & $P$ valuet & $\begin{array}{c}\text { Adjusted hazard ratio } \\
\text { model } 2 \ddagger\end{array}$ & $P$ value§ \\
\hline \multicolumn{6}{|l|}{ All cases } \\
\hline \multicolumn{6}{|l|}{ Systolic blood pressure: } \\
\hline All events and deaths & $1.06(1.03$ to 1.08$)$ & $1.04(1.02$ to 1.07$)$ & 0.002 & 1.05 (1.03 to 1.08$)$ & $<0.001$ \\
\hline All deaths & $1.06(1.03$ to 1.08$)$ & $1.04(1.02$ to 1.07$)$ & 0.003 & $1.06(1.03$ to 1.09$)$ & 0.001 \\
\hline Cardiovascular deaths & 1.05 (1.01 to 1.09$)$ & $1.03(0.99$ to 1.07$)$ & 0.145 & $1.05(1.01$ to 1.10$)$ & 0.050 \\
\hline \multicolumn{6}{|l|}{ Diastolic blood pressure: } \\
\hline All events and deaths & 1.05 (0.98 to 1.12$)$ & 1.05 (0.98 to 1.12$)$ & 0.192 & 1.09 (1.01 to 1.16$)$ & 0.024 \\
\hline All deaths & $1.00(0.92$ to 1.09$)$ & $1.01(0.92$ to 1.10$)$ & 0.910 & $1.04(0.95$ to 1.13$)$ & 0.460 \\
\hline Cardiovascular deaths & $1.00(0.88$ to 1.12$)$ & $1.00(0.88$ to 1.13$)$ & 0.934 & $1.04(0.91$ to 1.18$)$ & 0.600 \\
\hline \multicolumn{6}{|c|}{ No pre-existing cardiovascular disease } \\
\hline \multicolumn{6}{|l|}{ Systolic blood pressure: } \\
\hline All events and deaths & $1.06(1.04$ to 1.09$)$ & 1.05 (1.02 to 1.08$)$ & 0.001 & $1.05(1.02$ to 1.08$)$ & 0.001 \\
\hline All deaths & 1.06 (1.03 to 1.08$)$ & 1.04 (1.01 to 1.06$)$ & 0.020 & 1.05 (1.02 to 1.08$)$ & 0.008 \\
\hline Cardiovascular deaths & $1.06(1.02$ to 1.10$)$ & 1.03 (0.99 to 1.07$)$ & 0.179 & $1.05(1.00$ to 1.10$)$ & 0.115 \\
\hline \multicolumn{6}{|l|}{ Diastolic blood pressure: } \\
\hline All events and deaths & $1.03(0.95$ to 1.10$)$ & $1.03(0.95$ to 1.11$)$ & 0.503 & 1.06 (0.98 to 1.15$)$ & 0.143 \\
\hline All deaths & $0.97(0.88$ to 1.07$)$ & 0.98 (0.88 to 1.09$)$ & 0.680 & $1.02(0.91$ to 1.14$)$ & 0.770 \\
\hline Cardiovascular deaths & 0.97 (0.84 to 1.13$)$ & $0.98(0.84$ to 1.15$)$ & 0.812 & $1.00(0.85$ to 1.18$)$ & 0.997 \\
\hline
\end{tabular}

*Adjusted for Framingham 10 year cardiovascular risk score.

†P value for likelihood ratio test from omission of interarm differences from multivariable model 1.

$\ddagger$ Adjusted as for model 1 plus mean blood pressure, presence of diabetes, and pre-existing vascular disease (for all cases analyses).

$\S \mathrm{P}$ value for likelihood ratio test from omission of interarm differences from multivariable model 2. 
Table 5| Unadjusted and adjusted hazard ratios at predefined levels of interarm differences in blood pressure in participants without pre-existing vascular disease $(n=183)$

\begin{tabular}{|c|c|c|c|c|c|}
\hline Interarm difference & Unadjusted hazard ratio & Adjusted hazard ratio model $1^{*}$ & $P$ valuet & Adjusted hazard ratio model $2 \ddagger$ & $P$ value \\
\hline \multicolumn{6}{|c|}{ Combined cardiovascular and cerebrovascular events } \\
\hline \multicolumn{6}{|c|}{ Systolic blood pressure cut-off: } \\
\hline $10 \mathrm{~mm} \mathrm{Hg}$ & $3.5(2.1$ to 6.1$)$ & $3.1(1.8$ to 5.5$)$ & $<0.001$ & $3.0(1.7$ to 5.3$)$ & $<0.001$ \\
\hline $15 \mathrm{~mm} \mathrm{Hg}$ & $3.3(1.7$ to 6.3$)$ & $2.9(1.5$ to 5.5$)$ & 0.005 & $3.2(1.6$ to 6.4$)$ & 0.002 \\
\hline \multicolumn{6}{|c|}{ Diastolic blood pressure cut-off: } \\
\hline $10 \mathrm{~mm} \mathrm{Hg}$ & $2.1(0.9$ to 4.9$)$ & $2.1(0.9$ to 4.8$)$ & 0.129 & $3.2(1.2$ to 7.9$)$ & 0.032 \\
\hline \multicolumn{6}{|c|}{ Cardiovascular deaths } \\
\hline \multicolumn{6}{|c|}{ Systolic blood pressure cut-off: } \\
\hline $10 \mathrm{~mm} \mathrm{Hg}$ & $5.5(2.2$ to 13.8$)$ & $4.2(1.7$ to 10.8$)$ & 0.002 & $3.7(1.4$ to 9.9$)$ & 0.007 \\
\hline $15 \mathrm{~mm} \mathrm{Hg}$ & $3.1(1.1$ to 8.5$)$ & $2.2(0.8$ to 6.0$)$ & 0.170 & $2.8(1.0$ to 8.3$)$ & 0.079 \\
\hline \multicolumn{6}{|c|}{ Diastolic blood pressure cut-off: } \\
\hline $10 \mathrm{~mm} \mathrm{Hg}$ & $1.6(0.4$ to 6.8$)$ & $1.8(0.4$ to 8.0$)$ & 0.452 & $2.1(0.4$ to 11.0$)$ & 0.418 \\
\hline \multicolumn{6}{|l|}{ All deaths } \\
\hline \multicolumn{6}{|c|}{ Systolic blood pressure cut-off: } \\
\hline $10 \mathrm{~mm} \mathrm{Hg}$ & $3.4(1.9$ to 6.1$)$ & $2.8(1.5$ to 5.0$)$ & 0.001 & $2.6(1.4$ to 4.8$)$ & 0.002 \\
\hline $15 \mathrm{~mm} \mathrm{Hg}$ & $3.0(1.5$ to 6.0$)$ & $2.3(1.2$ to 4.6$)$ & 0.026 & $2.7(1.3$ to 5.4$)$ & 0.012 \\
\hline \multicolumn{6}{|c|}{ Diastolic blood pressure cut-off: } \\
\hline $10 \mathrm{~mm} \mathrm{Hg}$ & 0.7 (0.2 to 2.7$)$ & $0.8(0.2$ to 3.1$)$ & 0.679 & 1.1 (0.3 to 4.8$)$ & 0.891 \\
\hline \multicolumn{6}{|l|}{ Events or deaths } \\
\hline \multicolumn{6}{|c|}{ Systolic blood pressure cut-off: } \\
\hline $10 \mathrm{~mm} \mathrm{Hg}$ & $3.2(2.0$ to 5.0$)$ & $2.8(1.8$ to 4.5$)$ & $<0.001$ & $2.7(1.7$ to 4.4$)$ & $<0.001$ \\
\hline $15 \mathrm{~mm} \mathrm{Hg}$ & $2.8(1.7$ to 5.3$)$ & $2.6(1.5$ to 4.6$)$ & 0.003 & $2.8(1.6$ to 5.1$)$ & 0.002 \\
\hline \multicolumn{6}{|c|}{ Diastolic blood pressure cut-off: } \\
\hline $10 \mathrm{~mm} \mathrm{Hg}$ & $1.5(0.6$ to 3.4$)$ & $1.5(0.6$ to 3.4$)$ & 0.401 & $2.4(1.0$ to 5.9$)$ & 0.080 \\
\hline
\end{tabular}

*Adjusted for Framingham 10 year cardiovascular risk score.

†P value for likelihood ratio testing from omission of interarm differences from multivariable model 1.

$\ddagger$ Adjusted as for model 1 plus mean blood pressure and presence of diabetes.

$\S P$ value for likelihood ratio testing from omission of interarm differences from multivariable model 2. 


\section{Figures}

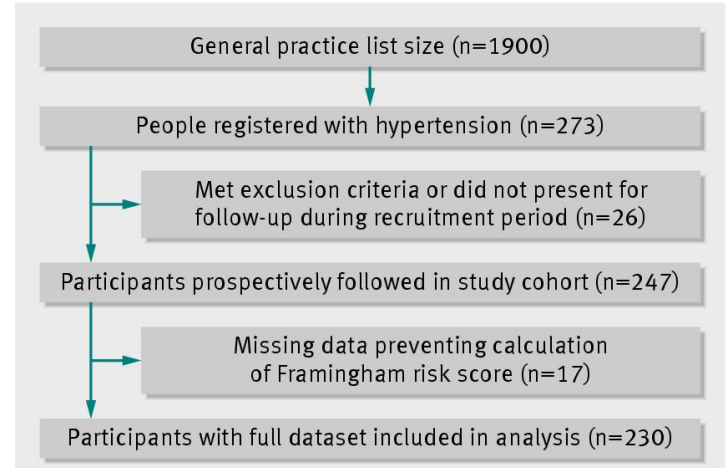

Fig 1 Flow of participants through study

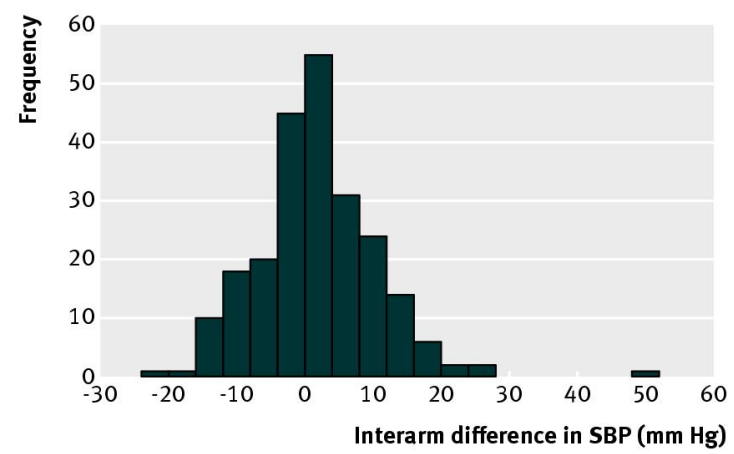

Fig 2 Distribution of interarm difference (mean of right arm minus mean of left arm) in systolic blood pressure (SBP) in study sample of 230 people with hypertension

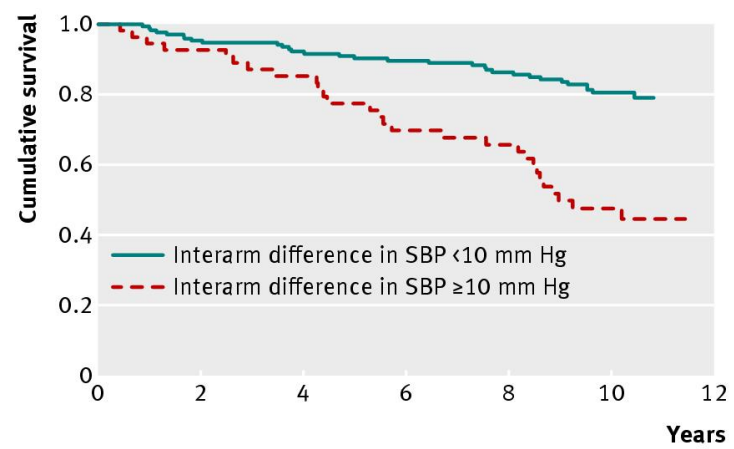

Fig 3 Kaplan-Meier plot for all cause mortality in 230 people with hypertension with or without an interarm difference in systolic blood pressure (SBP) of $\geq 10 \mathrm{~mm} \mathrm{Hg}$ 


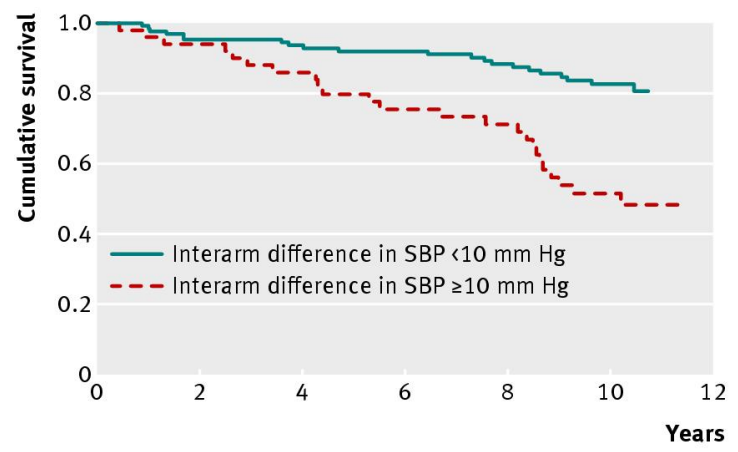

Fig 4 Kaplan-Meier plot for all cause mortality in 183 people with hypertension without cardiovascular disease at recruitment, with or without an interarm difference in systolic blood pressure (SBP) of $\geq 10 \mathrm{~mm} \mathrm{Hg}$

No pre-existing CVD

CVD risk $\ll 20 \%$ CVD risk $220 \%$ Interarm difference in SBP

$$
\begin{array}{lll}
---- & --- & \geq 10 \mathrm{~mm} \mathrm{Hg} \\
-- & & \geq 10 \mathrm{~mm} \mathrm{Hg}
\end{array}
$$

Existing CVD

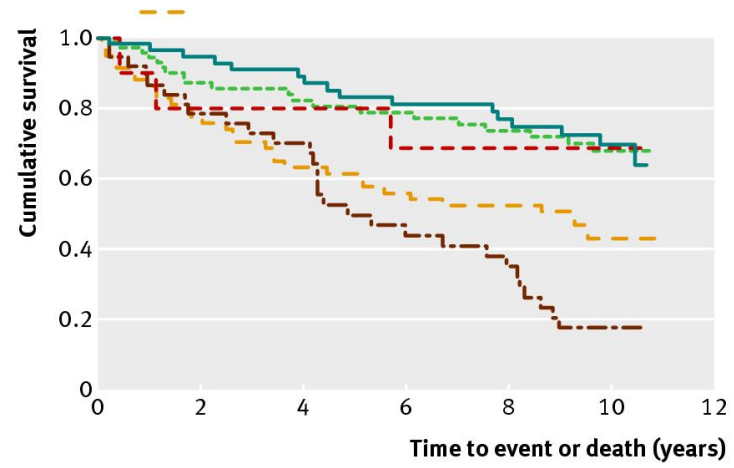

Fig 5 Kaplan-Meier survival curve for fatal and non-fatal events stratified by pre-existing cardiovascular disease (CVD) at recruitment, cardiovascular risk score, and interarm difference in systolic blood pressure (SBP) $\geq 10 \mathrm{~mm} \mathrm{Hg}(\mathrm{n}=230)$ 